\title{
PREVALENCE OF ERECTILE DYSFUNCTION AND CLINICAL CHARACTERISTICS IN PATIENTS WITH RHEUMATOLOGICAL DISEASES ASSISTED IN A RHEUMATOLOGY OUTPATIENT CLINIC
}

\author{
Weidson Henrique Lira Borges ${ }^{1}$, Mariana Souza Pessoa de Luna ${ }^{1}$, Hugo Deleon de Lima ${ }^{1}$, Thiago Henrique da Silva Rached ${ }^{1}$, \\ Mirella Patrício Rodrigues ${ }^{1}$, Filipe Tenório Lira Neto ${ }^{1}$, Laurindo Ferreira da Rocha Junior ${ }^{1, \star}$ \\ 1.Instituto de Medicina Integral Professor Fernando Figueira, Recife (PE), Brazil. \\ *Corresponding author: laurindorochajr@hotmail.com
}

\section{BACKGROUND}

Erectile dysfunction (ED) is a common condition that affects 20-50\% of men. Several studies have shown a higher prevalence of ED in patients with rheumatologic diseases (RDs). Furthermore, there is a positive association between ED and activity and disease severity, pain and fatigue in these patients. The purpose of this study was to determine the prevalence of ED in patients treated at a Rheumatology outpatient clinic in the Northeast of Brazil and to identify associations of ED with clinical parameters in these patients.

\section{METHODS}

Clinical-epidemiological questionnaire were carried out with 50 men with RDs and 46 age-matched controls between October of 2019 and September of 2020. Patients were clinically assessed and their medical records were reviewed. The presence of ED and quality of erection were assessed using the international index of erectile fuction-5 (IIEF-5) and the erection hardness score (EHS) questionnaires, respectively. Association measures were performed using the nonparametric Mann-Whitney test and Student's t-test. The Pearson and Spearmen tests were used to measure correlations. The GraphPad Prism 6.0 software was used to statistical analyses.

\section{RESULTS}

We studied 50 patients with 15 types of RDs with osteoarthritis (10), gout (8) and ankylosing spondylitis (7) being the most prevalent diseases. Erectile dysfunction was significatively higher in RD patients (76\%) compared to the control group (45.6\%). Erectile dysfunction was associated with older age $(p=0.0009)$, lower level of education $(p=0.0006)$, higher number of painful joints $(p=0.0001)$, Health assessment questionnaire (HAQ) $(p=0.0031)$ and triglycerides $(p=0.0026)$. Visual analogic scale (VAS) scores were higher in ED group compared with patients without ED for morning stiffness $(p=0.0023)$, current pain ( $p=0.0137)$, patient and physician global evaluation of disease ( $p=0.0018$ and 0.0217 , respectively). There were correlations between $I E F-5$ values with age $(r=-0.3562 ; p=0.0111)$, joint pain ( $r=-0.548 ; p<0.0001), \mathrm{HAQ}(r=-0.4987 ; p=0.0002)$ and values of VAS scales. The EHS also correlated with age $(r=-0.345 ; p=0.0141)$, joint pain $(r=-0.3685 ; p=0.0084), H A Q(r=-0.4201 ; p=0.0024)$, VAS for fatigue ( $r=-0.2883 ; p=0.0446)$, VAS for physician global evaluation of disease $(r=-0.347 ; p=0.0146)$, platelets $(n=31)$ $(r=0.3664 ; p=0.0426)$ and gamma glutamyl transferase $(n=11)(r=-0.5986 ; p=0.0280)$.

\section{CONCLUSION}

There is a high prevalence of ED in patients with RDs. According to our results, older patients with higher scores on joint pain and lower health status are at a greater risk of this condition. Erectile dysfunction is associated with worse clinical parameters in the sample studied.

\section{KEYWORDS}

Rheumatological diseases, Erectile dysfunction, Sexuality. 